\title{
Nano drug delivery system: An insight with review
}

\author{
Sanjeev Laller ${ }^{1}$, Divya Vardaini2,", Kanwalpreet Kaur ${ }^{3}$, Mamta Malik ${ }^{4}$, Vijay Kumar Vijayran $^{5}$ \\ ${ }^{1,4}$ Reader, Dept. of Oral Medicine and Radiology, PDM Dental College \& Research Institute, Bahadurgarh, Haryana, ${ }^{2}$ Post \\ Graduate Resident, Dept. of Pharmacology, Santosh Medical College, Ghaziabad, Uttar Pradesh, ${ }^{3}$ Lecturer, Dept. of \\ Conservative and Endodontics, BJS Dental College Ludhiana, Punjab, ${ }^{5}$ Dental Surgeon, Oscar Multispecialty Hospital and \\ Trauma Center, Rohtak, Haryana, India
}

*Corresponding Author:

Email: dvardaini@gmail.com

\begin{abstract}
Nanotechnology is the science of material featuring between 10-9 and 10-7 of a meter. Pharmaceutical nanotechnology is the latest advancement in pharmacology known for presenting multiple newer tools, opportunities and scope in drug delivery system having significant applications in disease diagnostics and treatment. Nano drug delivery system comprised of nano-sized products which can be transformed in numerous ways to improve their characteristics by leading to prolonged circulation, improved drug localization and enhanced drug efficacy. The aim of this paper is to summarize the types of pharma nano-systems and nanoparticles commonly used in drug delivery system along with their advantages, disadvantages and health issues related to nano drug delivery.
\end{abstract}

Keywords: Nanotechnology, Nano Drug Delivery, Nanoparticles, Liposomes.

\section{Introduction}

Latin word "dwarf" had given origin to word "Nano". Ideal size range offered by nanotechnology refers to one thousand millionth of a particular unit thus nanometer is one thousand millionth of a meter (i.e. 1 $\mathrm{nm}=10-9 \mathrm{~m}$ ). Newer invention of nanotechnology in biomedical and pharmaceutical science results in successful advantageous improvement of conventional means of drug delivery system (DDS) in various diseases. This technology enables the delivery of drugs that are poorly water soluble, bypass lever, increases oral bioavailability, increased stay in blood, increased tissue penetration and minimized side-effects with targeted tissues action. Background of nanotechnology in drug delivery reveals that around more than 150 years back, Michael Faraday prepared gold particles in nanometer scale. Later nano-liposomes and nanopolymer micelles were first prepared in 1960s followed by dendrimers in 1970s, though their relevance in nanotechnology was considered later. In 1980s micelles and in 1990s co-polymers of polyethylene glycol (PEG), PEG-Polylysine has been successfully invented as drug delivery system. More recently it was United States who contributed in modern nanotechnology by launching world first program in the field of nanotechnology. ${ }^{1-4}$

Nanoparticles (NPs) utilized in drug delivery are classified as organic and inorganic nanoparticles. Organic nanoparticles includes \{Polymers in DDS (polymeric miscalls, polymeric NPs, polymeric drug conjugates), dendrimers, nano crystals and lipid based NPs like liposomes, solid lipids $\}$ and Inorganic nanoparticles include \{Metal NPs (gold, silver, iron, platinum, quantum dots), Silica NPs (mesoporous, Xerogels) \}. As size of nanoparticles varies from different particle to particle and with relevance to method of preparation and techniques used. Particle size range for different nanotechnology techniques is shown in table 1 . This paper with an insight review regarding nano drug delivery system will summarize main nanoparticles used in drug delivery (carbon based structures, polymeric nanoparticles and liposomes) along with their advantages, disadvantages and health issues related to nanoparticles drug delivery and a brief note on nanoparticles and oral drug delivery. ${ }^{3,5,6}$

Table 1: Shows particle size range for different
nanotechnology techniques
\begin{tabular}{|l|c|}
\hline \multicolumn{1}{|c|}{ Techniques } & Size Range \\
\hline Nanoparticles & 10 to $1000 \mathrm{~nm}$. \\
\hline Liposomes & $15 \mathrm{~nm}$ to several $\mu \mathrm{m}$. \\
\hline Micelles & 10 to $80 \mathrm{~nm}$. \\
\hline Solid nano particles & 50 to $1000 \mathrm{~nm}$. \\
\hline Nanoemulsion & 50 to $1000 \mathrm{~nm}$. \\
\hline Lipid nanocapsule & Less than $100 \mathrm{~nm}$. \\
\hline Nanocrystals & 10 to $400 \mathrm{~nm}$ \\
\hline Carbon nanohorns & Less than $100 \mathrm{~nm}$. \\
\hline
\end{tabular}

Carbon Based Nanomaterials: The Carbon Nanotubes (CNT'S) (Fig. 1), Carbon Nano-diamonds and Carbon Nano-horns $(\mathrm{CNH})$ are used as carbon nano materials. Single-walled nanotubes (SWNTs) and multi-walled nanotubes (MWNTs) are most common and are arranged in fashion like a graphite sheet rolled up into a cylinder and capped at one or both ends by a buckyball having an excellent electronic and thermal conductivity. Due to their size and shape, carbon nanotubes can enter living cells without causing cell death or obvious damage. Carbon nanohorns have a structure similar to CNTs except they are closed at one end, forming a cone-shaped cap, or 'horn'. Drug immobilization in carbon based nano carriers is carried 
out in three ways: first by encapsulation of a drug in the carbon nanotube, secondly by chemical adsorption on the surface or in the spaces between the nanotubes (by electrostatic, hydrophobic, p-p interactions and hydrogen bonds), third being by attachment of active agents to functionalized carbon nanotubes (f-CNTs). When drugs are delivered using CNTs, results show increased solubility, low aggregation thus lower toxicity and increased anti-fungal action. The various drugs and immobilization method which uses in carbon nanomaterials include Gemcitabine (Encapsulation), Doxorubicin (Adsorption), Amphotericin B
(Conjugation), Cisplatin Encapsulation (capillary forces), Cisplatin-EGF (attachment to carbon nanotubes via amide linkages), Sulfamethoxazole (Adsorption) and Dexamethasone (Encapsulation). High length to diameter ratio and the toxicity of the sole material, which is graphite, are the main causative factor for adverse toxic effects. Carbon based nanomaterials are applicable in vaccine delivery, gene delivery, transport of peptides, nucleic acids and other drug molecules, gene silencing and to enhance the efficiency of diagnostic methods. ${ }^{2,3,6-8}$

\section{Fig. 1: Shows structure of carbon nano-tubes ${ }^{6}$.}

Polymeric Nanoparticles: Polymeric nanoparticles (PNPs) are colloidal solid particles with a size of diameter ranging from 10 to $100 \mathrm{~nm}$. Their structure can be spherical, branched or shell. They are developed from non-biodegradable and biodegradable polymers example being polyglycolic copolymers, poly [caprolactone, poly-acrylamide, poly-acrylate or natural polymers such as albumin, gelatin, alginate, collagen, DNA, chitosan, alginate. Methods of polymeric nanoparticles are solvent diffusion, emulsification diffusion, solvent evaporation, spontaneous emulsification and use of supercritical carbon dioxide and polymerization. Drug accumulation at target tissues is increased via using polymeric nanoparticles because their small sizes enable them to penetrate capillaries and to be taken up by cells and produce sustained effects of drugs for days to weeks. Drugs are incorporated into nanoparticles by dissolution, entrapment, adsorption, attachment or by encapsulation. Various drugs and treatment which uses polymeric NPs are Carboplatin (Ovarian, head, neck, and lung cancer), Doxorubicin (Wide spectrum of tumors), Lamivudine (Anti HIV/AIDS drug), Cisplatin (prostate cancer), Paclitaxel (Gliomas), Zidovudine (targeting lymphoid tissue), Amphotericin B (Neurodegenerative diseases), Vincristine + Verapamil (Hepatocellular carcinoma) and Clotrimazole (Antifungal drug). Polymeric nanoparticles are least toxic., ,, $9-11^{-1}$

Liposomes: Liposomes are small artificial vesicles (50 $-100 \mathrm{~nm}$ ) with an aqueous core surrounded by a lipid bilayer (Fig. 2) and lipid nanostructures can protect their contents from the conditions within the body that can result in potential degradation. They are developed from phospholipids like phosphatidylcholine, phosphatidylglycerol, phosphatidylethanolamine and phosphatidylserine. The liposomes are biocompatible, biodegradable, increase solubility of drugs; improve pharmacokinetic properties such as rapid metabolism, reduction of side effects, and increase of an in-vitro and in-vivo anticancer activity and therapeutic index of chemotherapeutic agents. Depending upon on their size and number of bilayers they are classified into three basic types: Multilamellar vesicles, small unilamellar vesicles (size $<100 \mathrm{~nm}$ ) and large unilamellar vesicles (size $>100 \mathrm{~nm}$ ). According to their physicochemical characteristics drug molecules can be entrapped in the aqueous space or intercalated into the lipid bilayer of liposome. Encapsulation incorporates drug into liposomes and their release from liposomes is influenced by the liposome composition, $\mathrm{pH}$, osmotic gradient, and the surrounding environment. A variety of biological and drug compounds have been delivered using liposomes. Liposomes and their respective model drugs include Amiloride hydrochloride used in cystic fibrosis (Small molecular liposome), Budesonide in asthma (Small molecular liposome), Insulin in diabetes (Protein liposome), Interleukin-2 in lung cancer (Protein liposome), Tobramycin in pulmonary infections (Small molecular liposome) and Ketotifen in asthma (Small molecular liposome). Other similar vesicular structures include transferosomes, ethosomes, niosomes and marinosomes which are used mainly for transdermal delivery. ${ }^{2,3,6,11-13}$ 


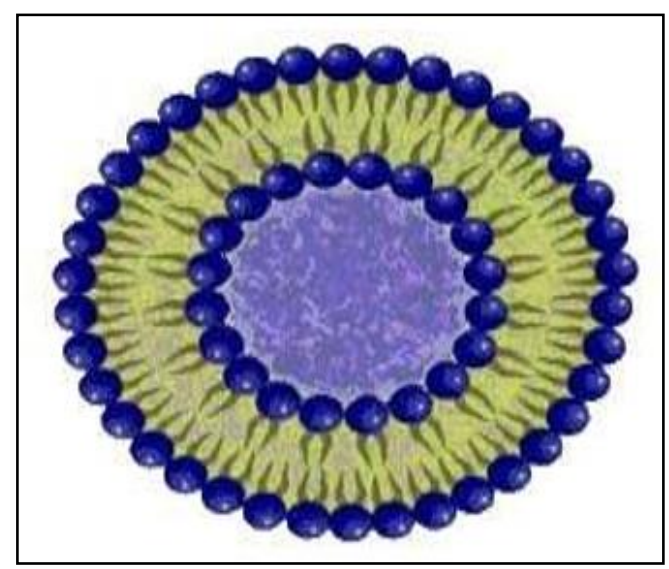

Fig. 2: Shows bilayer structure of liposome ${ }^{6}$

Nanoparticles and Oral Drug Delivery System: Oral administration is the oldest and the commonest mode of drug administration because it is safer, more convenient, does not need assistance, non-invasive and painless route. But drugs that are poorly permeable or easily degradable in the gastrointestinal tracts (GIT) like peptides and proteins create challenge as their absorption is affected by barriers like epithelial cell lining, the mucus layer, proteolytic enzymes in the gut lumen (such as pepsin, trypsin and chymotrypsin), and proteolytic enzymes (endopeptidases) resulting in low bioavailability. In this case drug loaded in nanoparticles is prevented from enzymatic degradation and results in enhanced absorption at intestinal lymphatic tissues containing Mcells (the Peyer's patches). Also many polymers can be used in the nanoparticle composition to provide sustained release in oral cavity. ${ }^{14}$

Advantages of Nano drug delivery: ${ }^{6,15,16}$

1. Drug protection from degradation.

2. Reduction in required doses.

3. Insoluble drugs can be delivered easily.

4. Tumors allow an enhanced permeability and retention effect.

5. Drug targeting is possible with nano drug system.

6. Long drug circulation in blood.

7. Improve the oral bioavailability of the agents that are not effectively used orally.

8. Toxicity is less.

9. Smaller drug dosage form.

10. Faster dissolution of drug.

11. Drugs can be delivered through oral, nasal, parenteral, intra-ocular etc.

12. Ability to control and sustain the drug before reaching the specific site of action

Disadvantages of Nano drug delivery: $:^{15,17}$

1. Due to altered physical properties nano-particles get aggregated.

2. Nano-particles are highly reactive because of smaller particle size and larger surface area.

3. Drug loading is less due to small size of nanoparticles.
4. Health Hazards: Free nanoparticles exhibit serious health threat since they are more difficult to contain due to airborne and can be inhaled. Example- nanoparticles cause inflammation and subsequent pulmonary tumors compared with larger sized particles having identical chemical composition.

\section{Conclusion}

Nano drug delivery systems dramatically improve the pharmacological and therapeutic properties of conventional drugs like reduced drug dosage, improvised in the absorption of the drug, deliver the drug to the right place in the living system, increased local concentration of the drug at the desired site with least side effects. Multifunctional nanoparticles hold great promise for the future of multiple disease treatment but they also pose some health issues and other challenges. Nanotechnology can be developed in future to treat all type of diseases in human at the same time invest in resources to determine how best to manage nanotechnology safely and ethically.

\section{References}

1. Martin CR. Welcome to nanomedicine. Nanomedicine 2006;1(1):5.

2. Wilczewska AZ, Niemirowicz K, Markiewicz KH, Car H. Pharmacological Reports 2012;64:1020-37.

3. Wanigasekara J and Witharana C. Applications of Nanotechnology in Drug Delivery and Design - An Insight. Current Trends in Biotechnology and Pharmacy 2016;10(1):78-91.

4. Zhang Y., Chan H.F. and Leong K.W. Advanced Materials and Processing for Drug Delivery: The Past and the Future. Adv Drug Deliv Rev 2013;65(1):104-20.

5. Safari, J. and Zarnegar Z. Advanced drug delivery systems Nanotechnology of health design. J Saudi Chemi Soci 2013:18:85-99.

6. Banveer J et al. Nanotechnology: A safe and effective drug delivery system. Asi J Pharmace and Clini Res 2010;3(3):159-65.

7. Reilly RM. Carbon nanotubes: potential benefits and risks of nanotechnology in nuclear medicine. J Nucl Med. 2007;48:1039-42.

8. Fu T et al. Nanotechnology in Therapeutics. A Focus on Nanoparticles as a Drug Delivery System. Nanomedicine 2012;7(8):1253-71.

9. Ochekpe NA, Olorunfemi PO and Ngwuluka NC. Nanotechnology and Drug Delivery Part 2: Nanostructures for Drug Delivery. Tropi J Pharmace Res 2009;8(3):275-87.

10. Arias JL, Ruiz MA, López-viota M, Delgado AV. Poly (alkylcyanoacrylate) colloidal particles as vehicles for anti-tumour drug delivery: a comparative study. Colloids and Surfaces B: Biointerfaces 2008;62:64-70.

11. Saad MZH, Jahan R, Bagul U. Nanopharmaceuticals: a New perspective of drug delivery system. Asi J Biomed Pharm Sci.

12. M R and Sahin N O, Manufacturing methods and mechanism of formation of lipid vesicles. In: Nanoliposomes: From 2012;2:14.

13. Mozafari Fundamentals to Recent Developments. Mozafari MR \& Mortazavi S M (Eds.), Trafford Publishing Ltd, Oxford, UK 2005:39-48. 
14. Ramaldes G.A. et al. Study of the pilot production process of long-circulating and $\mathrm{pH}$-sensitive liposomes containing cisplatin. J Liposome Res 2011;21:60-69.

15. Shingai Majuru and Moses O. Oyewumi.

Nanotechnology in Drug Development and Life Cycle Management. M.M. de Villiers et al. (eds.),

Nanotechnology in Drug Delivery 2009; Chapter 20:597619.
16. Sriharitha, Preethi J, Swaroop H. A Review on Nanoparticles in Targeted Drug Delivery System. JOMS 2016;4(4):1-6.

17. Duggal D. Role of Nanotechnology in New Drug Delivery Systems. Int J Drug Dev \& Res 2011;3(4):4-8.

18. Lee KP, Kelly DP, Oneal FO, Kennedy GL. Lung response to ultrafine kertar aramid synthetic fibrils following 2-year inhalation exposure in rats. Findam Appl Toxicol. 1998;11:1-20. 